\title{
A Method for Solving a Class of Boundary Value Problems of Laguerre Equation
}

\author{
Li Ren ${ }^{1}$, Shunchu Li $^{1, *}$, Dongdong Gui ${ }^{2}$ \\ ${ }^{1}$ School of science, Xihua University, Chengdu, China \\ ${ }^{2}$ Beijing Dongrunke Petroleum Technology Co.,Ltd., Beijing 100029, China \\ *Corresponding author: lishunchu@163.com
}

Received April 10, 2015; Revised April 23, 2015; Accepted April 29, 2015

\begin{abstract}
Based on the analysis of the boundary value problem of Laguerre equation, this paper studies the similar structure of its solution expression. This paper is found that its solution can be obtained by combining similar kernel function with coefficients of left boundary condition. While the similar kernel function is constructed by both the function of guide solution and coefficients of right boundary condition. Hence, we proposed a method for solving this class of boundary value problems: the similar constructing method.
\end{abstract}

Keywords: Laguerre equation, boundary value problem, similar kernel function, the similar constructing method

Cite This Article: Li Ren, Shunchu Li, and Dongdong Gui, "A Method for Solving a Class of Boundary Value Problems of Laguerre Equation.” American Journal of Applied Mathematics and Statistics, vol. 3, no. 3 (2015): 89-92. doi: 10.12691/ajams-3-3-1.

\section{Introduction}

As is known to all, we often involve solving differential equations in practical problems. Studying the inherent law of the solutions to differential equations plays a crucial role for simplifying the solving process. Then, can the form of the solutions( the solutions belong to the various differential equations in the same boundary value problem) be represented by using a uniform formula? Since 2004, the definite answer has been made in many relevant reports. At the same time, the solutions of some secondorder homogeneous linear ordinary differential equations [1-9], the solutions of some second-order homogeneous linear partial differential equations [10,11], and the solutions of the seepage equations in some reservoir engineering [12,13,14] were studied, with gradually forming the similar constructing theory and similar constructing method of the solutions that belong to differential equations.

Laguerre equation has some comprehensive applications in several research areas, such as physics and engineering. Based on the above study, this paper will study a class of boundary value problem (BVP)of the Laguerre equation as follows:

$$
\left\{\begin{array}{l}
x y^{\prime \prime}+(1-x) y^{\prime}+n y=0 \quad x \in(a, b) \\
{\left[E y+(1+E F) y^{\prime}\right]_{x=a}=D} \\
{\left[K y+H y^{\prime}\right]_{x=b}=0}
\end{array}\right.
$$

where $D, E, F, K, H, a, b, n$ are real constants, $D \neq 0$, $K^{2}+H^{2} \neq 0,0<a<b, n$ is a positive constant.

\section{Preliminary Knowledge}

Lemma 1 General solution to Laguerre equation can be expressed as[15]:

$$
y=D_{1} L_{n}(x)+D_{2} G(-n, 1, x)
$$

Where $D_{1}, D_{2}$ are arbitrary constants, $L_{n}(x)$ is the Laguerre polynomial, and $G(-n, 1, x)$ is the second class of Kummer functions.

Lemma 2 Known that $F(\alpha, \gamma, \chi)$ is the first class of Kummer functions, it can get the conclusion as follows [15]:

$$
\frac{d}{d x} F(\alpha, \gamma, \chi)=\frac{\alpha}{\gamma} F(\alpha+1, \gamma+1, \chi)
$$

where

$$
\begin{aligned}
& F(\alpha, \gamma, \chi)=\sum_{k=0}^{\infty} \frac{(\alpha)_{k}}{k !(\gamma)_{k}} \chi^{k}, \\
& (\alpha)_{k}=\alpha(\alpha+1) \cdots(\alpha+k-1) .
\end{aligned}
$$

According to the lemma 2, we can be obtained as follows:

$$
\begin{gathered}
\frac{d}{d x} L_{n}(x)=\frac{d}{d x}(n ! F(-n, 1, x))=n !(-n) F(1-n, 2, x), \\
\frac{d}{d x} G(-n, 1, x)=\frac{d}{d x}[F(-n, 1, x) \ln x-1] \\
=\ln x(-n) F(1-n, 2, x)+\frac{1}{x} F(-n, 1, x) .
\end{gathered}
$$




\section{The Main Theorem and Its Proof}

The probative thought in this paper is that the similar construction of the solution of the BVP (1) is obtained by extending from the special case of right boundary condition to the general situation. Now, this paper discusses the BVP (1) with the special case of right boundary condition.

Theorem 1 If the boundary value problem

$$
\left\{\begin{array}{l}
x y^{\prime \prime}+(1-x) y^{\prime}+n y=0 \quad x \in(a, b) \\
{\left[E y+(1+E F) y^{\prime}\right]_{x=a}=D} \\
y(b)=0, \text { 或 } y^{\prime}(b)=0
\end{array}\right.
$$

has one solution, then the similar construction of the solution can be expressed as:

$$
y(x)=\frac{D}{E+\frac{1}{F+\psi(a)}} \frac{1}{F+\psi(a)} \psi(x)
$$

And where $\psi(x)$ is kernel function:

$$
\psi(x)=\left\{\begin{array}{l}
\frac{\phi_{1}(x)}{\phi_{1}^{\prime}(a)}, y(b)=0 \\
\frac{\phi_{2}(x)}{\phi_{2}{ }^{\prime}(a)}, y^{\prime}(b)=0
\end{array}\right.
$$

Where

$$
\begin{aligned}
& \phi_{1}(x)=G(-n, 1, b) L_{n}(x)-L_{n}(b) G(-n, 1, x), \\
& \phi_{2}(x)=G^{\prime}(-n, 1, b) L_{n}(x)-L_{n}{ }^{\prime}(b) G(-n, 1, x) .
\end{aligned}
$$

Proof. The basic equation in left and right regions of the BVP (1) is Laguerre equation, according to the lemma 1 , we can obtain the general solution of (1) as follows:

$$
y=D_{1} L_{n}(x)+D_{2} G(-n, 1, x)
$$

where $D_{1}, D_{2}$ are undetermined constants.

Substituting Eq.(6) into the left boundary condition of the BVP(3), yields:

$$
\begin{aligned}
& {[E n ! F(-n, 1, a)+(1+E F) n !(-n) F(1-n, 2, a)] D_{1}} \\
& +\left[\begin{array}{l}
F(-n, 1, a) \ln a-1 \\
+\ln a(-n) F(1-n, 2, a)+\frac{1}{a} F(-n, 1, a)
\end{array}\right] D_{2}=D
\end{aligned}
$$

1. when the right boundary condition of the $\mathrm{BVP}(3)$ for $y(b)=0$, then Substituting Eq.(6) into it :

$$
\begin{aligned}
& D_{1} n ! F(-n, 1, b)+D_{2} G(-n, 1, b) \\
& =D_{1} n ! F(-n, 1, b)+D_{2}[F(-n, 1, b) \ln b-1]=0
\end{aligned}
$$

Incorporating Eqs.(7)and(8), we can acquire the expressions about coefficients of Eq.(6) as follows:

$$
\begin{aligned}
D_{1} & =\frac{D(-G(-n, 1, b))}{M_{1} G(-n, 1, b)-M_{2} L_{n}(b)} \\
D_{2} & =\frac{D L_{n}(b)}{M_{1} G(-n, 1, b)-M_{2} L_{n}(b)}
\end{aligned}
$$

Substituting Eqs.(9) and (10) into Eq.(6), we can gain the solution:

$$
y(x)=\frac{D\left[G(-n, 1, b) L_{n}(x)-L_{n}(b) G(-n, 1, x)\right]}{M_{1} G(-n, 1, b)-M_{2} L_{n}(b)}
$$

where

$$
\begin{aligned}
& M_{1}=E L_{n}(a)+(1+E F) L_{n}{ }^{\prime}(a), \\
& M_{2}=E G(-n, 1, a)+(1+E F) G^{\prime}(-n, 1, a) .
\end{aligned}
$$

Let

$$
\psi(x)=\frac{\phi_{1}(x)}{\phi_{1}{ }^{\prime}(a)}=\frac{G(-n, 1, b) L_{n}(x)-L_{n}(b) G(-n, 1, x)}{G(-n, 1, b) L_{n}{ }^{\prime}(a)-L_{n}(b) G^{\prime}(-n, 1, a)} .
$$

After simplifying, then

$$
y(x)=\frac{D}{E+\frac{1}{F+\psi(a)}} \frac{1}{F+\psi(a)} \psi(x)
$$

2. when the right boundary condition of the BVP (3) for $y^{\prime}(b)=0$, then Substituting Eq.(6) into it:

$$
\begin{aligned}
& D_{1} n !(-n) F(1-n, 2, b) \\
& +D_{2}\left[\ln b(-n) F(1-n, 2, b)+\frac{1}{b} F(-n, 1, b)\right]=0
\end{aligned}
$$

Incorporating Eqs.(7) and (13), we can acquire the expressions about coefficients of Eq.(6) as follows:

$$
\begin{aligned}
& D_{1}=\frac{D\left(-G^{\prime}(-n, 1, b)\right)}{M_{1} G^{\prime}(-n, 1, b)-M_{2} L_{n}{ }^{\prime}(b)} \\
& D_{2}=\frac{D L_{n}^{\prime}(b)}{M_{1} G^{\prime}(-n, 1, b)-M_{2} L_{n}^{\prime}(b)}
\end{aligned}
$$

Substituting Eqs.(14) and(15)into Eq.(6), we can gain the solution:

$$
y(x)=\frac{D\left[G^{\prime}(-n, 1, b) L_{n}(x)-L_{n}{ }^{\prime}(b) G(-n, 1, x)\right]}{M_{1} G^{\prime}(-n, 1, b)-M_{2} L_{n}{ }^{\prime}(b)}
$$

where

$$
\begin{aligned}
& M_{1}=E L_{n}(a)+(1+E F) L_{n}^{\prime}(a), \\
& M_{2}=E G(-n, 1, a)+(1+E F) G^{\prime}(-n, 1, a) .
\end{aligned}
$$

Let

$$
\begin{aligned}
& \psi(x)=\frac{\phi_{2}(x)}{\phi_{2}{ }^{\prime}(a)} \\
& =\frac{G^{\prime}(-n, 1, b) L_{n}(x)-L_{n}{ }^{\prime}(b) G(-n, 1, x)}{G^{\prime}(-n, 1, b) L_{n}{ }^{\prime}(a)-L_{n}{ }^{\prime}(b) G^{\prime}(-n, 1, a)} .
\end{aligned}
$$

After simplification, we can gain the solution as same as Eq.(12).

After discussing the structure of solution of the BVP(4), that is the special situation of the BVP(1). Now, we return to the theme in this paper--the BVP(1)that includes the general situation of the right boundary condition, and study the BVP(1) by the above way.

Theorem 2 If the BVP(1) has one solution, then the similar structure of the solution can be expressed as: 


$$
y(x)=\frac{D}{E+\frac{1}{F+\psi(a)}} \frac{1}{F+\psi(a)} \psi(x)
$$

And where $\psi(x)$ is kernel function:

$$
\psi(x)=\frac{K \phi_{1}(x)+H \phi_{2}(x)}{K \phi_{1}{ }^{\prime}(a)+H \phi_{2}{ }^{\prime}(a)}
$$

Proof. The basic equation in left and right regions of the BVP (1) is Laguerre equation, according to the lemma 1, we can obtain the general solution of (1) as follows:

$$
y=D_{1} L_{n}(x)+D_{2} G(-n, 1, x)
$$

where $D_{1}, D_{2}$ are undetermined constants.

when the right boundary condition of the BVP(1) for $\left[K y+H y^{\prime}\right]_{X=b}=0$, then substituting Eq.(18) into it:

$$
\begin{aligned}
& K\left[D_{1} n ! F(-n, 1, b)+D_{2} G(-n, 1, b)\right] \\
& +H\left[D_{1} L_{n}{ }^{\prime}(b)+D_{2} G^{\prime}(-n, 1, b)\right]=0
\end{aligned}
$$

Substituting Eq.(18) into the left boundary condition of the BVP(1), yields:

$$
\begin{aligned}
& {\left[\begin{array}{l}
E n ! F(-n, 1, a) \\
+(1+E F) n !(-n) F(1-n, 2, a)
\end{array}\right] D_{1}} \\
& +\left[\begin{array}{l}
F(-n, 1, a) \ln a-1 \\
+\ln a(-n) F(1-n, 2, a)+\frac{1}{a} F(-n, 1, a)
\end{array}\right] D_{2}=D
\end{aligned}
$$

Incorporating Eqs.(19)and(20), we can acquire the expressions about coefficients of Eq.(18) as follows:

$$
\begin{gathered}
D_{1}=\frac{-D Y_{1}}{M_{1} Y_{1}-M_{2} Y_{2}} \\
D_{2}=\frac{D Y_{2}}{M_{1} Y_{1}-M_{2} Y_{2}}
\end{gathered}
$$

Substituting Eqs.(21) and(22)into Eq.(18), we can gain the solution of the BVP(1):

$$
y(x)=\frac{D\left[Y_{2} G(-n, 1, X)-Y_{1} L_{n}(x)\right]}{M_{1} Y_{1}-M_{2} Y_{2}}
$$

Where

$$
\begin{aligned}
& Y_{1}=K G(-n, 1, b)+H G^{\prime}(-n, 1, b), \\
& Y_{2}=K L_{n}(b)+H L_{n}{ }^{\prime}(b), \\
& M_{1}=E L_{n}(a)+(1+E F) L_{n}{ }^{\prime}(a), \\
& M_{2}=E G(-n, 1, a)+(1+E F) G^{\prime}(-n, 1, a) .
\end{aligned}
$$

Let

$$
\psi(x)=\frac{K \phi_{1}(x)+H \phi_{2}(x)}{K \phi_{1}{ }^{\prime}(a)+H \phi_{2}{ }^{\prime}(a)} .
$$

After simplification, we can gain the solution as same as Eq.(16).

\section{The Ideology and Steps of the Similar Constructing Method}

To observe and analyze theorems 1 and 2, it is not hard to find :

1) Through observing Eqs.(5) and (6), we can obtain the similar kernel function $\psi(x)$ which consists of $\phi\left(x_{1}, x_{2}\right)=G\left(-n, 1, x_{2}\right) L_{n}\left(x_{1}\right)-L_{n}\left(x_{2}\right) G\left(-n, 1, x_{1}\right)$ an d coefficients $K$ 、 $H$ of right boundary condition $\left[K y+H y^{\prime}\right]_{X=b}=0$, as follows:

$$
\psi(x)=\frac{K \phi_{1}(x)+H \phi_{2}(x)}{K \phi_{1}{ }^{\prime}(a)+H \phi_{2}{ }^{\prime}(a)}
$$

where

$$
\begin{aligned}
& \phi\left(x_{1}=x, x_{2}=b\right)=\phi_{1}(x) \\
& =G(-n, 1, b) L_{n}(x)-L_{n}(b) G(-n, 1, x), \\
& \left.\frac{\partial}{\partial x_{2}} \phi\left(x_{1}, x_{2}\right)\right|_{x_{2}=b}=\phi_{2}(x) \\
& =G^{\prime}(-n, 1, b) L_{n}(x)-L_{n}{ }^{\prime}(b) G(-n, 1, x) .
\end{aligned}
$$

2) According to theorem 2, we can know the solution of BVP(1) by combining the coefficients $E, F, D$ of left boundary condition $\left[E y+(1+E F) y^{\prime}\right]_{x=a}=D$.

From the above, it is not difficult to gain a method, which is called the similar constructing method, for solving a class of boundary value problems of Laguerre equation. And it is easy to induce steps of the similar constructing method. The concrete steps are as follows:

Step 1 constructing binary function $\phi\left(x_{1}, x_{2}\right)$

We structure binary function $\phi\left(x_{1}, x_{2}\right)$ by combining the second class of Kummer function $G(-n, 1, x)$ and the Laguerre polynomial $L_{n}(x)$, and that $G(-n, 1, x)$ and $L_{n}(x)$ are linearly independent. Then, we can base $\phi_{1}(x)$ and $\phi_{2}(x)$ on lemma 2 .

\section{Step 2 constructing the similar kernel function $\psi(x)$}

The similar kernel function $\psi(x)$ can be structured by using binary function $\phi\left(x_{1}, x_{2}\right)$ and coefficients $K$ 、 $H$ of right boundary condition $\left[K y+H y^{\prime}\right]_{X=b}=0$, that is to say, the equation(17). If the right boundary condition is $y(b)=0$ (i.e. $K=1, H=0$ ) or $y^{\prime}(b)=0$ (i.e. $K=0$, $H=1$ ), it can respectively combine binary function $\phi\left(x_{1}, x_{2}\right)$ to obtain the corresponding similar kernel function, in other words, the equation(5).

Step 3 obtaining the solution to the BVP $y(x)$

To the BVP(1), the solution can be obtained by assembling coefficients $D, E, F$ of left boundary condition $\left[E y+(1+E F) y^{\prime}\right]_{x=a}=D$, that is, the equation (16).

\section{The Application of the Similar Constructing Method}

Solving the boundary value problem as follows: 


$$
\left\{\begin{array}{l}
x y^{\prime \prime}+(1-x) y^{\prime}+y=0 \quad x \in(a, b) \\
y^{\prime}(x)_{x=1}=1 \\
2 y^{\prime}(x)_{x=2}=0
\end{array}\right.
$$

Comparing with the boundary value problem (1) and (26), we know that $n=1$,

$E=0, a=1, D=1, K=0, H=2, b=2 \quad$. The basic equation in left and right regions of the BVP (26) is Laguerre equation, then the two linear independent solutions of the main equation is $L_{1}(x)$ and $G(-1,1, x)$. According to steps of the similar constructing method, we solve the boundary value problem(26).

Step 1 constructing binary function $\phi\left(x_{1}, x_{2}\right)$

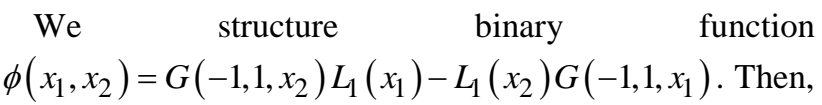
according to lemma 2 and $\operatorname{Eqs}(24) \sim(25)$, we can obtain $\phi_{1}(x)$ and $\phi_{2}(x)$, as follows:

$$
\begin{aligned}
& \phi_{1}(x)=G(-1,1,2) L_{1}(x)-L_{1}(2) G(-1,1, x), \\
& \phi_{2}(x)=G^{\prime}(-1,1,2) L_{1}(x)-L^{\prime}(2) G(-1,1, x) .
\end{aligned}
$$

\section{Step 2 constructing the similar kernel function $\psi(x)$}

According to Eqs.(17),we can structure the similar kernel function $\psi(x)$, as the follow:

$$
\psi(x)=\frac{0 \times \phi_{1}(x)+2 \cdot \phi_{2}(x)}{0 \times \phi_{1}{ }^{\prime}(1)+2 \cdot \phi_{2}{ }^{\prime}(1)}=\frac{\phi_{2}(x)}{\phi_{2}{ }^{\prime}(1)}
$$

\section{Step 3 obtaining the solution to the BVP $y(x)$}

According to Eqs.(16), the boundary value problem(26) can be obtained respectively as the follow:

$$
\begin{aligned}
y(x) & =\psi(x)=\frac{\phi_{2}(x)}{\phi_{2}^{\prime}(1)} \\
& =\frac{G^{\prime}(-1,1,2) L_{1}(x)-L_{1}^{\prime}(2) G(-1,1, x)}{G^{\prime}(-1,1,2) L_{1}^{\prime}(1)-L_{1}^{\prime}(2) G^{\prime}(-1,1,1)}
\end{aligned}
$$

Where

$$
\begin{aligned}
& G^{\prime}(-1,1,2)=-\ln 2 F(0,2,2)+\frac{1}{2} F(-1,1,2), \\
& L_{1}(x)=F(-1,1, x) \\
& G^{\prime}(-1,1,1)=F(-1,1,1), \\
& G(-1,1, x)=F(-1,1, x) \ln x-1 \\
& F(0,2,2)=\sum_{k=0}^{\infty} \frac{(0)_{k}}{k !(2)_{k}} 2^{k}=0, \\
& F(-1,1, x)=\sum_{k=0}^{\infty} \frac{(-1)_{k}}{k !(1)_{k}} x^{k} .
\end{aligned}
$$

\section{Conclusions}

The Eq.(16), that is the solution of the boundary value of Laguerre equation, can be obtained by assembling coefficients of left boundary condition and similar kernel function. It can be expressed by one uniform formula.
In the research process, we know that we only need to change the corresponding similar kernel functions to obtain the solution to the BVP (1) when the right boundary condition of the BVP (1) changes. The method is simple and effective for solving the boundary value of differential equation.

\section{Acknowledgment}

This work is supported the by the Scientific Research Fund of the Sichuan Provincial Education Department of China (Grant No. 12ZA164).

\section{References}

[1] Zongrong Cheng, Shunchu Li. The Similar Structure Method Solving the Boundary Value Problem of Bessel Equations (in Chinese)[J]. Journal of Sichuan Normal University (Natural Science), 2011,34(6): 850-853.

[2] Junchao Wang, Shunchu Li. The Similar Structure of Solutions of Second-Order Linear Homogeneous Differential Equation (in Chinese)[J]. Pure Mathematics, 2012, 2(1): 23-27.

[3] Shunchu Li, Zhi-Jian Liao. Constructing the Solution of Boundary Value Problem of the Differential Equation with it's an Arbitrary Non-trivial Solution (in Chinese) [J]. Journal of Sichuan University (Natural Science Edition), 2012,49(6):1209-1213.

[4] Rongjun Huang, Shunchu Li, Dongxu Xu. Similar Structured Method of Solution to Boundary Value of First Weber Equation (in Chinese)[J]. Journal of Mianyang Normal University, 2012, 31(11):1-5(-15).

[5] Xitao Bao, Shunchu Li, ZhiJian Liao. Similar Constructive Method of Solution for the Boundary Value Problem of Euler Hyper-geometric Differential Equation (in Chinese)[J]. Journal of Southwest University of Science and Technology, 2012, 27(4):101-105.

[6] Shunchu Li. The Similarity Structuring Method of Boundary Value Problems of the Composite Differential Equations (in Chinese) [J]. Journal of Xihua University (Natural Science Edition), 2013, 32(4): 27-31.

[7] Congyin Fan, Shunchu Li, Dongxu Xu. Similar Structure Method of Solution of a class of Thomson Equation Boundary Value Problem (in Chinese)[J]. Advances in Applied Mathematics, 2012, 1(2): 41-48.

[8] Fu-rong Wang, Shunchu Li, Dongxu Xu. The Similarity Construction Method of a Kind of Boundary-Value Problem of Airy Equation(in Chinese) [J]. Journal of Hubei Normal University (Natural Science), 2013, 33(1):79-85.

[9] Zhijian Liao, Shunchu Li. A new method for solving the boundary value problem of extend Bessel equation: The similar structuring method [J].Journal of University of Science and Technology of China, 2013,43(12):975-979.

[10] Shunchu Li. The Formal Similarity of Solutions in the Laplace Space on the Class of Partial Differential Equation System (in English)[J]. Journal of Xihua University (Natural Science Edition), 2007, 26(4):83-86.

[11] Minhui Jia, Shunchu Li. The Formal Similarity of Solutions in the Laplace Space on the Class of Fluid Flow Differential Equation [J]. Journal of Electronic Science and Technology of China, 2005, 3(2):172-174.

[12] Shunchu Li, Jianjun Zhang. Similar Structure of Pressure Distribution in the Fractal Dual Porosity Reservoir (in Chinese)[J]. Journal of Xihua University (Natural Science Edition), 2006, 25(1):40-43.

[13] Li Xu, Shunchu Li, Cuicui Sheng. Similar Structure of Flow Effective Well Radius Model through Homogeneous Reservoir Solutions (in Chinese)[J]. Journal of Chongqing Normal University (Natural Science Edition), 2011, 28(4):32-34.

[14] Xuxia Xiao, Shunchu Li. The Similar Structure of Solutions for the Boundary Value Problem of Euler's Hypergeometric Differential Equation (in Chinese)[J]. Journal of Inner Mongolia Normal University (Natural Science Edition), 2012, 41(6): 597600(-603).

[15] Shishi Liu, Shida Liu. The special function[M]. Beijing: China Meteorological Press, 2002. 